\title{
Analysis of ECRH Pre-Ionization for Plasma Start-Up in JT-60SA ${ }^{*)}$
}

\author{
Kazuyoshi HADA, Kazunobu NAGASAKI ${ }^{1)}$, Kai MASUDA ${ }^{1)}$, Ryota KINJO, Shunsuke IDE ${ }^{2)}$ \\ and Akihiko ISAYAMA ${ }^{2)}$ \\ Graduate School of Energy Science, Kyoto University, Uji, Kyoto 611-0011, Japan \\ 1) Institute of Advanced Energy, Kyoto University, Uji, Kyoto 611-0011, Japan \\ 2) Japan Atomic Energy Agency, Naka, Ibaraki 311-0193, Japan
}

(Received 9 December 2011 / Accepted 31 May 2012)

\begin{abstract}
Plasma start-up assisted by electron cyclotron resonance heating (ECRH) pre-ionization is theoretically studied for the superconducting tokamak JT-60SA by using a zero-dimensional (0-D) model. Because the toroidal electric field is limited to $0.5 \mathrm{Vm}^{-1}$, we clarify the conditions for a robust plasma initiation. Five temporal equations are solved for a spatially-uniform plasma: the electron and neutral density equations, the electron and ion energy density equations, and the electric circuit equation. The numerical calculation results show that an absorbed ECRH power of $150 \mathrm{~kW}$ is required to start up the plasma, assuming an initial neutral density of $3.0 \times 10^{18} \mathrm{~m}^{-3}$, and that reducing the oxygen and carbon impurity densities facilitates successful start-up. We discuss the dependence of the absorbed ECRH power on the neutral density and impurities. The simulation qualitatively reproduces experimental results from JT-60U.
\end{abstract}

(C) 2012 The Japan Society of Plasma Science and Nuclear Fusion Research

Keywords: pre-ionization, electron cyclotron resonance heating, superconducting tokamak, JT-60SA, zerodimensional model

DOI: $10.1585 /$ pfr.7.2403104

\section{Introduction}

Plasma is initiated by a loop voltage generated by solenoid coils in tokamaks. In superconducting tokamaks, which aim at steady-state operation, the loop voltage generated for plasma breakdown is lower than that of normal conducting tokamaks due to a limited current ramp rate and thick vacuum vessel wall. Therefore it is difficult to start up plasmas if the radiation loss is large, that is, if the vacuum chamber wall is not in good condition. To ensure reliable plasma breakdown, pre-ionization using electron cyclotron resonance heating (ECRH) has been proposed, and successful start-up with ECRH at a low loop voltage has been demonstrated in normal conducting tokamaks such as JT-60U [1] and DIII-D [2] and in the superconducting tokamak KSTAR [3]. In the International Thermonuclear Experimental Reactor (ITER), the toroidal electric field is limited to $0.3 \mathrm{Vm}^{-1}$, and pre-ionization using $170 \mathrm{GHz}$ ECRH is planned for plasma start-up [4]. Because the JT60SA superconducting tokamak, which is now under construction at the JAEA [5], also has a limited toroidal electric field $\left(0.5 \mathrm{Vm}^{-1}\right)$, we need to clarify the conditions for robust plasma initiation. Application of the second harmonic $110 \mathrm{GHz}$ ECRH is under consideration for reliable start-up. However, it is not yet clear how much ECRH power is necessary for plasma start-up and how plasma conditions such as impurities affect the plasma start-up.

A zero-dimensional (0-D) model that describes

author's e-mail: hada.880@iae.kyoto-u.ac.jp

*) This article is based on the presentation at the 21st International Toki Conference (ITC21).
ECRH-assisted start-up in ISX-B was proposed by Kulchar et al. [6], and more advanced models were developed by Lloyd et al. [7] and Bae et al. [8]. Lloyd et al. included the effects of impurities, and Bae et al. considered the error field effects and poloidal field coil circuit equations. In this paper, we study plasma start-up assisted by ECRH pre-ionization in JT-60SA (major radius, $R_{0}=2.97 \mathrm{~m}$; minor radius, $a=1.18 \mathrm{~m}$; and toroidal magnetic field, $B_{\mathrm{T}}=$ $2.25 \mathrm{~T}$ ) by using a $0-\mathrm{D}$ model. The model consists of the electron and neutral (hydrogen atom) density equations, the electron and ion (hydrogen ion) energy density equations, and the electric circuit equation. The main purposes of this paper are to examine the conditions for reliable start-up in JT-60SA and clarify the dominant physical process in ECRF plasma initiation.

This paper is organized as follows. The 0-D model is described in Sec.2, and the calculation results are discussed in Sec. 3. The conclusion is presented in Sec. 4.

\section{Zero-Dimensional (0-D) Model}

The ECRH pre-ionization effects are theoretically investigated by a $0-\mathrm{D}$ model to examine the conditions for a reliable start-up in JT-60SA and to understand the physical process. The 0-D model consists of five temporal equations: the electron and neutral density equations, the electron and ion energy density equations, and the electric circuit equation. These equations are solved for spatiallyuniform plasma. In this model, we assume that the major and minor radii remain constant and all of the injected 
ECRH power is absorbed.

The electron density $n_{\mathrm{e}}$ is given by

$$
\frac{\mathrm{d} n_{\mathrm{e}}}{\mathrm{d} t}=S n_{0} n_{\mathrm{e}}-\frac{n_{\mathrm{e}}}{\tau_{\mathrm{p}}}
$$

where $n_{0}$ is the neutral density, the particle confinement time $\tau_{\mathrm{p}}$ is considered as $100 \mathrm{~ms}$, and $S$ is the coefficient rate for electron ionization, given by

$$
S=\frac{2 \times 10^{-13}}{6+T_{\mathrm{e}} / 13.6} \sqrt{\frac{T_{\mathrm{e}}}{13.6}} \exp \left(\frac{-13.6}{T_{\mathrm{e}}}\right) .
$$

$T_{\mathrm{e}}$ is the electron temperature in electronvolts.

We assume that the total number of particles are conserved,

$$
\frac{\mathrm{d} n_{0}}{\mathrm{~d} t}=-\frac{\mathrm{d} n_{\mathrm{e}}}{\mathrm{d} t}
$$

The impurity density assumed to be much smaller than the hydrogen density.

The electron and ion temperatures are determined by the electron and ion energy density equation,

$$
\begin{aligned}
\frac{\mathrm{d} U_{\mathrm{e}}}{\mathrm{d} t}= & p_{\mathrm{ECRH}}+p_{\mathrm{OH}}-p_{\mathrm{IONIZ}}-p_{\mathrm{BREM}} \\
& \quad-p_{\mathrm{IRAD}}-p_{\mathrm{EQU}}-U_{\mathrm{e}}\left(v_{\mathrm{err}}+v_{\mathrm{dr}}+v_{\mathrm{E}}\right), \\
\frac{\mathrm{d} U_{\mathrm{i}}}{\mathrm{d} t}= & p_{\mathrm{EQU}}-p_{\mathrm{CX}}-U_{\mathrm{i}} v_{\mathrm{E}}
\end{aligned}
$$

where $U_{\mathrm{e}}=3 n_{\mathrm{e}} T_{\mathrm{e}} / 2$ is the electron energy density and $U_{\mathrm{i}}$ $=3 n_{\mathrm{i}} T_{\mathrm{i}} / 2$ is the ion energy density. Here $p_{\mathrm{ECRH}}$ is the absorbed ECRH power density. Because the electron density and temperature vary with time, the ECRH power absorption efficiency can be considered in future. The ohmic heating power density $p_{\mathrm{OH}}$ is given by $p_{\mathrm{OH}}=I_{\mathrm{p}}^{2} R_{\mathrm{p}} / V_{\mathrm{p}}$, where $R_{\mathrm{p}}$ is the plasma resistance, and $V_{\mathrm{p}}$ is the plasma volume.

The ionization and radiation loss $p_{\text {IONIZ }}$ is given by

$$
p_{\text {IONIZ }}=\frac{V_{\mathrm{n}}}{V_{\mathrm{p}}} n_{0} n_{\mathrm{e}} S W_{\mathrm{D}},
$$

where $V_{\mathrm{n}}$ is the volume occupied by neutrals, and $W_{\mathrm{D}}$, the total energy lost per ionization, is considered to be $30 \mathrm{eV}$. The bremsstrahlung power loss $p_{\text {BREM }}$ is given by

$$
p_{\text {BREM }}=1.53 \times 10^{-38} n_{\mathrm{e}} T_{\mathrm{e}}^{1 / 2}\left(n_{\mathrm{D}}+\sum_{I} n_{I}\left\langle Z^{2}\right\rangle_{I}\right),
$$

where $n_{\mathrm{D}}$ is the hydrogen ion density, and $\left\langle Z^{2}\right\rangle_{I}$ is the mean-square charge.

The line radiation loss through the interaction between electrons and impurity ions $p_{\mathrm{IRAD}}$ is given by

$$
p_{\text {IRAD }}=n_{\mathrm{e}} n_{I} \times 10^{f},
$$

where $f=-33.93+4.888 Q-2.432 Q^{2}+0.3697 Q^{3}$ for the carbon impurity and $f=-34.06+4.194 Q-1.827 Q^{2}$
$+0.2467 Q^{3}$ for oxygen impurities with $Q=\log T_{\mathrm{e}}$. The impurity density $n_{I}$ is treated as $n_{I}=f_{I} n_{\mathrm{e}}$, where $f_{I}$ is the ratio of the impurities to the electron density.

The equipartition loss transferred from electrons to ions $p_{\mathrm{EQU}}$ is given by

$$
\begin{aligned}
p_{\mathrm{EQU}}= & 7.75 \times 10^{-34}\left(T_{\mathrm{e}}-T_{\mathrm{i}}\right) \frac{n_{\mathrm{e}} \ln \Lambda}{T_{\mathrm{e}}^{3 / 2}} \\
& \times\left(\frac{n_{\mathrm{D}}}{2}+\sum_{I} \frac{n_{I}\left\langle Z^{2}\right\rangle_{I}}{A_{I}}\right),
\end{aligned}
$$

where $\ln \Lambda$ is the Coulomb logarithm, and $A_{I}$ is the impurity ion mass number.

The charge exchange loss between hydrogen atoms and hydrogen ions $p_{\mathrm{CX}}$ is given by

$$
p_{\mathrm{CX}}=\frac{V_{\mathrm{n}}}{V_{\mathrm{p}}} e n_{\mathrm{D}} n_{0}\left(T_{\mathrm{i}}-T_{0}\right) S_{\mathrm{CX}},
$$

where $T_{0}$ is the temperature of background neutrals, and $S_{\mathrm{CX}}$ is the rate coefficient for charge exchange,

$$
S_{\mathrm{CX}}=1.066 \times 10^{-14} T_{\mathrm{i}}^{0.327} .
$$

For the detailed formulae, see [7,8]. In the initial phase, the error field causes direct connection of the field lines to the vacuum chamber wall; consequently, the electrons escape from the plasma along the lines. The error field loss rate $v_{\text {err }}$ is given by

$$
v_{\mathrm{err}}=\left(\frac{\delta B}{B}\right)\left(\frac{V_{T}}{a}\right)\left[1-\frac{I_{\mathrm{p}}}{I_{\mathrm{c}}}\right]
$$

for $I_{\mathrm{p}}<I_{\mathrm{C}}$ and by $v_{\mathrm{err}}=0$, for $I_{\mathrm{p}}>I_{\mathrm{C}}$, where $V_{\mathrm{T}}$ is the thermal velocity of electrons, and $I_{\mathrm{C}}$ is the critical current, $I_{\mathrm{C}}=4 \pi a \delta B / \mu_{0}$. The drift loss rate due to $\operatorname{grad} B$ drift and curvature drift [9], $\nu_{\mathrm{dr}}$, is given by

$$
v_{\mathrm{dr}}=4.95 \times 10^{-9} \frac{T_{\mathrm{e}}}{a R_{0} B} .
$$

Here $v_{\mathrm{E}}$ is the energy loss rate given by the empirical law based on "neo-Alcator scaling".

The electric circuit equation is given by

$$
\frac{\mathrm{d} I_{\mathrm{p}}}{\mathrm{d} t}=\frac{V_{\mathrm{L}}-I_{\mathrm{p}} R_{\mathrm{p}}}{L}
$$

where $V_{\mathrm{L}}$ is the applied loop voltage, and $L$ is the plasma inductance,

$$
L=\mu_{0} R_{0}\left(\ln \frac{8 R_{0}}{a}-1.75\right)
$$

Although the plasma inductance is a strong function of the current profile and varies rapidly with time as the current channel forms, for simplicity, we assume a flat current profile and treat the plasma inductance as a constant. The plasma inductance is $L=4.67 \mu \mathrm{H}$ for $R_{0}=2.97 \mathrm{~m}$ and $a$ $=1.18 \mathrm{~m}$.

These equations were numerically solved by the fourth-order Runge-Kutta method. 


\section{Simulation Results}

\subsection{Simulation results for JT-60SA}

We calculated plasma start-up using the 0-D model with the JT-60SA parameters. Figure 1 shows the temporal behavior of the loop voltage and absorbed ECRH power. The ECRH power was turned on at $t=0.0 \mathrm{~s}$; that is, it was applied before the loop voltage is turned on. The loop voltage of approximately $9 \mathrm{~V}$, which corresponds to $0.5 \mathrm{Vm}^{-1}$, was applied from $t=0.05 \mathrm{~s}$ to $0.15 \mathrm{~s}$. We scanned the initial electron temperature and density in the range of $T_{\mathrm{e}}=$ $0.1-2.0 \mathrm{eV}$, from $n_{\mathrm{e}}=1.0 \times 10^{15}$ to $1.0 \times 10^{17} \mathrm{~m}^{-3}$ and confirmed that these changes have little impact on the temporal behavior of the main variables. We assume the following initial parameters: an electron density of $1.0 \times 10^{16} \mathrm{~m}^{-3}$, a neutral density of $3.0 \times 10^{18} \mathrm{~m}^{-3}$, electron and ion tem-

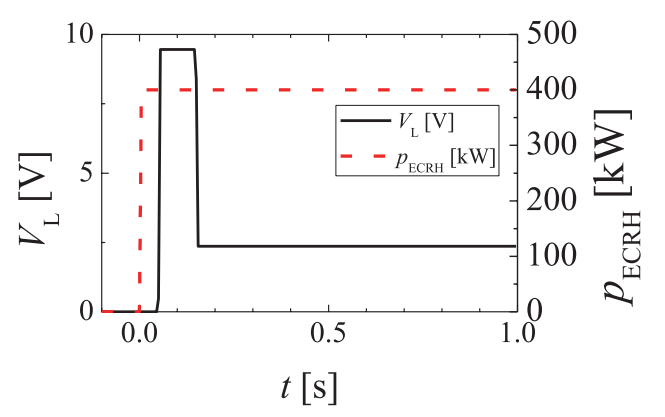

Fig. 1 Temporal behavior of the loop voltage and the absorbed ECRH power.

(a)

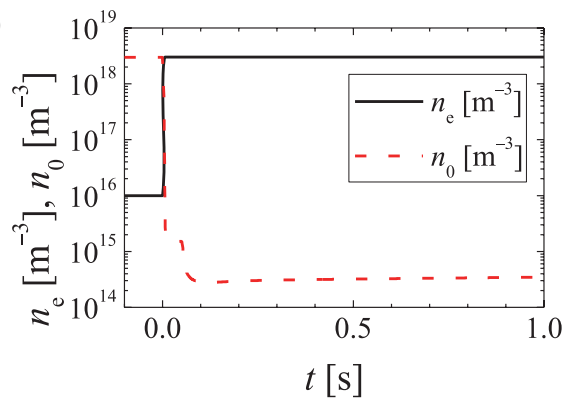

(b)

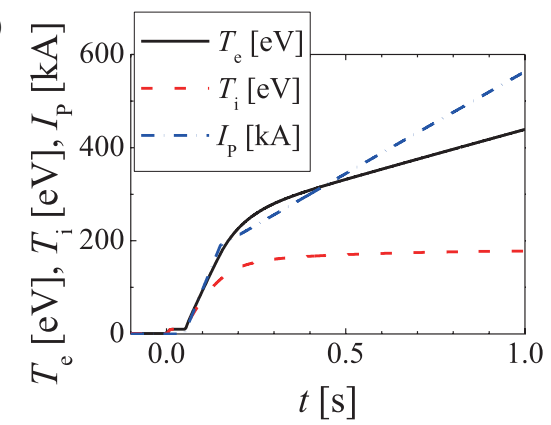

Fig. 2 (a) Time evolution of electron and neutral densities for a successful start-up. Absorbed ECRH power is $p_{\mathrm{ECRH}}=$ $400 \mathrm{~kW}$. (b) Time evolution of electron and ion temperatures and current for a successful start-up. Absorbed ECRH power is $p_{\mathrm{ECRH}}=400 \mathrm{~kW}$. peratures of $T_{\mathrm{e}}=1.0 \mathrm{eV}$ and $T_{\mathrm{i}}=1.0 \mathrm{eV}$, respectively, carbon and oxygen densities of $0.1 \%$ of the electron density, and an absorbed ECRH power of $p_{\mathrm{ECRH}}=400 \mathrm{~kW}$. In this paper, we define successful start-up as occurring when the electron temperature becomes sufficiently high, so that $p_{\text {IRAD }}$ is no longer a large fraction of the total power loss from the plasma. This generally applies when $T_{\mathrm{e}}>100 \mathrm{eV}$. Figure 2 shows the temporal behavior of the electron and neutral densities, the electron and ion temperatures, and the plasma current in a successful start-up. The electron density rises promptly to near $3.0 \times 10^{18} \mathrm{~m}^{-3}$, and the neutral density decreases with increasing electron density. These electrons are produced by pre-ionization using ECRH. In contrast, when the ECRH power is reduced, the discharge fails. Figure 3 shows the temporal behavior of the electron and neutral densities, electron and ion temperatures, and plasma current in an unsuccessful start-up. In the initial phase, the line radiation power due to impurity ions, ionization power, and equipartition power are the dominant losses in the electron energy density equation. In this case, the plasma cannot overcome the radiation barrier. The electron energy density is much smaller than that in successful start-up, and the electron temperature remains low.

Figure 4 shows the dependence of the electron temperature and plasma current at $t=1.0 \mathrm{~s}$ on the absorbed ECRH power. The initial conditions are an electron density of $1.0 \times 10^{16} \mathrm{~m}^{-3}$ and a neutral density of $3.0 \times 10^{18} \mathrm{~m}^{-3}$. The carbon and oxygen densities are the same as that

(a)

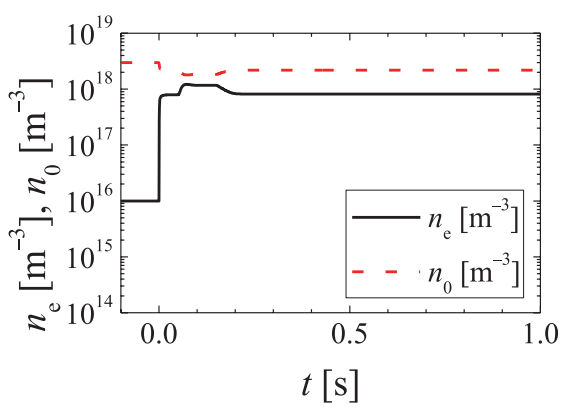

(b)

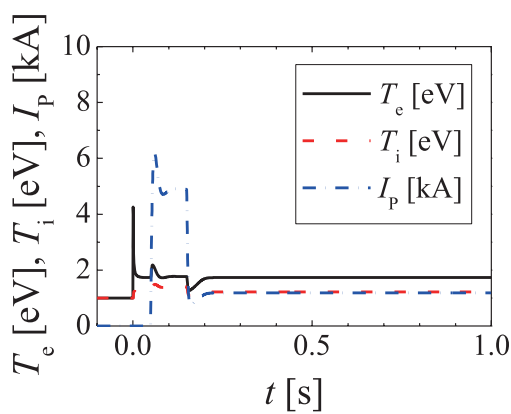

Fig. 3 (a) Time evolution of electron and neutral densities for an unsuccessful start-up. Absorbed ECRH power is $p_{\mathrm{ECRH}}=$ $100 \mathrm{~kW}$. (b) Time evolution of electron and ion temperature and plasma current for an unsuccessful start-up. Absorbed ECRH power is $p_{\mathrm{ECRH}}=100 \mathrm{~kW}$. 


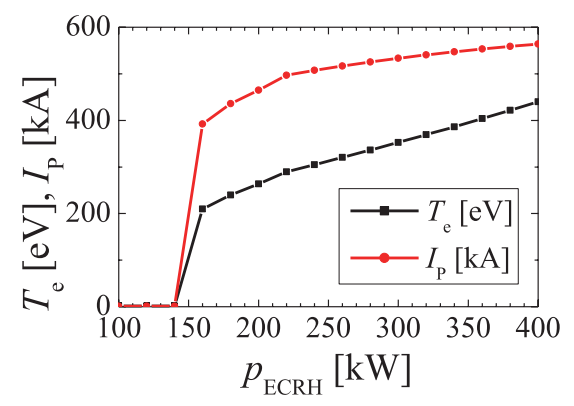

Fig. 4 Electron temperature and plasma current at $t=1.0 \mathrm{~s}$.

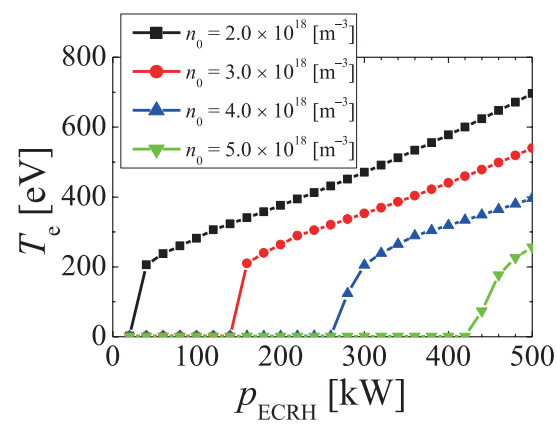

Fig. 5 Electron temperature at $t=1.0 \mathrm{~s}$ for a neutral density of $2.0-5.0 \times 10^{18} \mathrm{~m}^{-3}$.

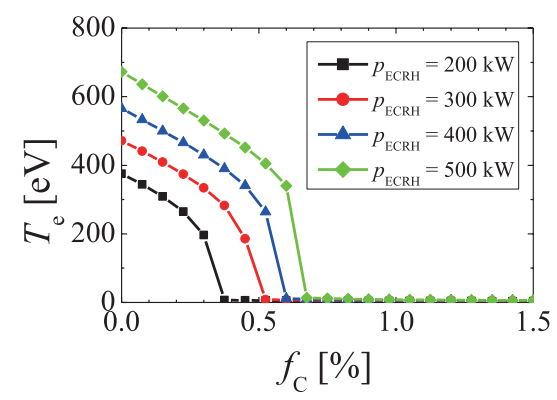

Fig. 6 Electron temperature as a function of the fraction of carbon impurities for an absorbed ECRH power of 200$500 \mathrm{~kW}$.

shown in Fig. 2. The figure reveals a threshold in ECRH power for successful start-up; an ECRH power of approximately $150 \mathrm{~kW}$ is required when the initial neutral density is $3.0 \times 10^{18} \mathrm{~m}^{-3}$.

Figure 5 shows the electron temperature at $t=1.0 \mathrm{~s}$ versus the absorbed ECRH powers. The initial neutral density was scanned from $2.0 \times 10^{18} \mathrm{~m}^{-3}$ to $5.0 \times 10^{18} \mathrm{~m}^{-3}$. More ECRH power is required as the initial neutral density increases because the radiation power loss increases. Below the threshold power, the radiation barrier cannot be overcome, and the electron temperature remains as low as $1 \mathrm{eV}$. Impurities have an important role in plasma start-up. Figures 6 and 7 show the electron temperature at $t=1.0 \mathrm{~s}$ for various ratios of impurities to the electron density. The ratios of carbon and oxygen impurities to the electron density were varied between $0.0 \%$ and $1.5 \%$. The effect of car-

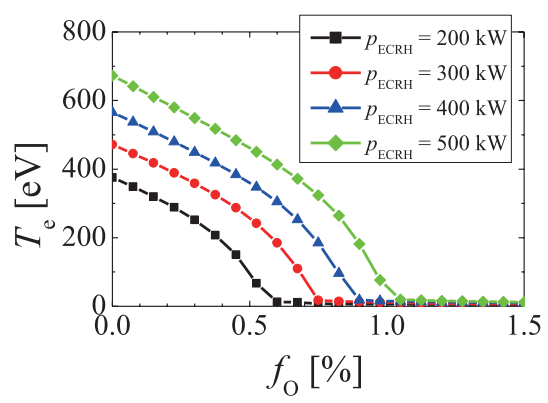

Fig. 7 Electron temperature as a function of the fraction of oxygen impurities for an absorbed ECRH power of 200-500 kW.

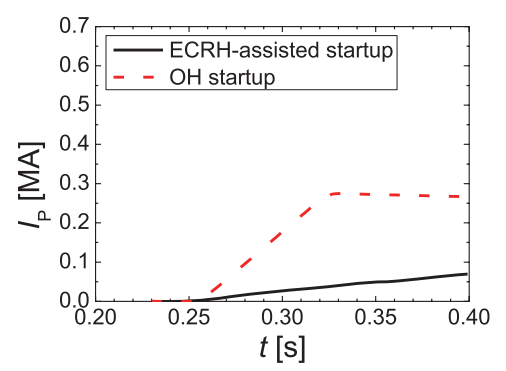

Fig. 8 Temporal behavior of the plasma current for JT-60U.

bon impurities is slightly larger than that of oxygen impurities. When the carbon impurities were more than $0.5 \%$ of the electron density, the required ECRH power was more than $300 \mathrm{~kW}$. Suppression of oxygen and carbon impurities would be useful to ensure successful start-up and reduce the required ECRH power.

\subsection{Comparison with JT-60U experimental results}

To validate the 0 -D model used in this paper, we calculated the plasma start-up in JT-60U by using the 0-D model and compared it with the experimental results. We assumed a major radius of $R_{0}=3.05 \mathrm{~m}$, a minor radius of $a$ $=0.72 \mathrm{~m}$, a self-inductance of $5.49 \mu \mathrm{H}$, an initial electron density of $1.0 \times 10^{16} \mathrm{~m}^{-3}$, and no impurities. Two types of start-ups were calculated: ECRH-assisted start-up with an ECRH power of $950 \mathrm{~kW}$ (from $t=0.23 \mathrm{~s}$ ), a loop voltage of $4 \mathrm{~V}(t=0.25-0.35 \mathrm{~s})$, and an initial neutral density of $0.64 \times 10^{18} \mathrm{~m}^{-3}$; and $\mathrm{OH}$ start-up with a loop voltage of $25 \mathrm{~V}(t=0.25-0.33 \mathrm{~s})$ without ECRH power, and with an initial neutral density of $1.4 \times 10^{18} \mathrm{~m}^{-3}$. Figure 8 shows the calculated temporal behavior of the plasma current. In the ECRH-assisted start-up, the plasma current increased to nearly $0.07 \mathrm{MA}$ at $0.4 \mathrm{~s}$. In the $\mathrm{OH}$ start-up, the plasma current increased to nearly $0.26 \mathrm{MA}$ at $0.4 \mathrm{~s}$. The numerical simulation qualitatively reproduced the time evolution of the experimental results [1] in both cases. However, these currents are approximately half of those in the experimental results described in [1]. A self-inductance of $3.0 \mu \mathrm{H}$ can reproduce the experimental results. However, it 
is not yet clear whether this is a realistic value because we have not considered the effects of the eddy current or the radial profile in the $0-\mathrm{D}$ model. A one dimensional model considering the radial structure is now under development for more quantitative estimation.

\section{Conclusion}

We investigated the effects of ECRH pre-ionization on plasma start-up using the JT-60SA parameters and a 0-D model. The calculation shows that the ECRH power is effective for plasma start-up under low loop voltage conditions. An ECRH power of $150 \mathrm{~kW}$ is required to start up the plasma for $n_{\mathrm{e}}=3.0 \times 10^{18} \mathrm{~m}^{-3}$. There is a threshold in ECRH power for a successful start-up. The threshold depends on the initial neutral density, and greater ECRH power is required as the initial neutral density increases. In addition, it depends on the carbon and oxygen impurity densities because the radiation loss increases with increasing impurity densities. This implies that for a reliable startup, it is important to control the neutral density and reduce the impurity densities. The calculation using the JT-60U parameters qualitatively reproduced the time evolution of the experimental results. For a more quantitative and detailed analysis, the $0-\mathrm{D}$ model will be extended to a 1-D model.

\section{Acknowledgement}

This work was partially supported by collaboration research of JT-60SA.

[1] K. Kajiwara et al., Nucl. Fusion. 45, 694 (2005).

[2] G.L. Jackson et al., Nucl. Fusion. 47, 257 (2007).

[3] Y.S. Bae et al., Nucl. Fusion. 49, 022001 (2009).

[4] Y. Gribov et al., Nucl. Fusion. 47, S385 (2007).

[5] S. Ishida et al., Nucl. Fusion. 51, 094018 (2011).

[6] A.G. Kulchar et al., Phys. Fluids 27, 7 (1984).

[7] B. Lloyd et al., Plasma Phys. Control. Fusion. 38, 1627 (1996).

[8] Y.S. Bae et al., IEEE Trans. Plasma Sci. 31, 4 (2003).

[9] F.F. Chen, Introduction to Plasma Physics and Controlled Fusion, 2nd ed (New York: Plenum, 1984, vol.1) p. 43. 